\title{
Homogeneous polar foliations of complex hyperbolic spaces
}

\author{
Jürgen Berndt and José CARlos Díaz-Ramos
}

We prove that, up to isometric congruence, there are exactly $2 n+$ 1 homogeneous polar foliations of the complex hyperbolic space $\mathbb{C} H^{n}, n \geq 2$. We also give an explicit description of each of these foliations.

\section{Introduction}

A proper isometric action of a connected Lie group $G$ on a complete Riemannian manifold $M$ is called polar if there exists a connected closed submanifold $\mathcal{S}$ of $M$ such that

(i) $\mathcal{S}$ meets each orbit of the action, i.e., for each $p \in M$ the intersection of $\mathcal{S}$ and the orbit $G \cdot p$ of $G$ containing $p$ is nonempty;

(ii) $\mathcal{S}$ intersects each orbit orthogonally, i.e., for all $p \in \mathcal{S}$ the tangent space $T_{p} \mathcal{S}$ of $\mathcal{S}$ at $p$ is contained in the normal space $\nu_{p}(G \cdot p)$ of $G \cdot p$ at $p$.

Such a submanifold $\mathcal{S}$ is called a section of the action. The classical paper on this topic is by Dadok [5] who determined all connected Lie subgroups of the orthogonal group $O(n)$ acting polarly on the Euclidean space $\mathbb{E}^{n}$. Using Dadok's result it is not difficult to deduce a classification of all polar actions up to orbit equivalence on the sphere $S^{n}$ and in the real hyperbolic space $\mathbb{R} H^{n}$, see e.g., [14]. Podestà and Thorbergsson classified in [13] all polar actions on compact Riemannian symmetric spaces of rank one up to orbit equivalence. Kollross studied polar actions on compact Riemannian symmetric spaces of higher rank and obtained a complete classification in [9] for the case when the isometry group of the symmetric space is simple, and in [10] for the case when the symmetric space is an exceptional compact Lie group. A striking difference between the rank one case and the higher rank case is that in the higher rank case polar actions are hyperpolar, that is, the section $\mathcal{S}$ is flat. This is not true for the rank one case.

The classification of polar actions on Riemannian symmetric spaces of noncompact type is more complicated due to the noncompactness of the 
isometry groups. One cannot expect a general correspondence using the concept of duality between symmetric spaces of compact type and of noncompact type. It is known that there are symmetric spaces of compact type which do not admit any polar actions, whereas on every symmetric space of noncompact type there are polar actions. However, for actions by reductive groups Kollross established in [11] a correspondence. Polar actions with a fixed point on Riemannian symmetric spaces of noncompact type have been classified by the second author and Kollross in [6]. Polar actions with codimension one orbits, or equivalently, cohomogeneity one actions, on Riemannian symmetric spaces of noncompact type are also well understood, see $[2,3]$.

The motivation for this paper is to understand better the general classification problem for the complex hyperbolic space $\mathbb{C} H^{n}=S U(1, n) / S(U(1)$ $U(n))$. We restrict here to the situation when the orbits of the action form a foliation of $\mathbb{C} H^{n}$, in which case we call the foliation a homogeneous polar foliation of $\mathbb{C} H^{n}$. There are two trivial homogeneous polar foliations on $\mathbb{C} H^{n}$, one for which the leaves are points in $\mathbb{C} H^{n}$, and one with exactly one leaf equal to $\mathbb{C} H^{n}$. Consider a restricted root space decomposition $\mathfrak{g}=\mathfrak{g}_{-2 \alpha} \oplus \mathfrak{g}_{-\alpha} \oplus \mathfrak{g}_{0} \oplus \mathfrak{g}_{\alpha} \oplus \mathfrak{g}_{2 \alpha}$, where $\mathfrak{g}$ is the Lie algebra of $S U(1, n)$. The subspace $\mathfrak{g}_{0}$ decomposes into $\mathfrak{g}_{0}=\mathfrak{k}_{0} \oplus \mathfrak{a}$, where $\mathfrak{a}$ is a one-dimensional subspace of $\mathfrak{p}$ in a Cartan decomposition $\mathfrak{g}=\mathfrak{k} \oplus \mathfrak{p}$ and $\mathfrak{k}_{0}$ is the centralizer of $\mathfrak{a}$ in $\mathfrak{k}$. The Kähler structure of $\mathbb{C} H^{n}$ induces a complex structure on $\mathfrak{g}_{\alpha}$, turning $\mathfrak{g}_{\alpha}$ into an $(n-1)$-dimensional complex vector space. To each real subspace $\mathfrak{w}$ of $\mathfrak{g}_{\alpha}$ and each subspace $V$ of $\mathfrak{a}$ we associate a subalgebra $\mathfrak{s}_{V, \mathfrak{w}}$ of $\mathfrak{g}$. Note that $\operatorname{dim} V \in\{0,1\}$ and $\operatorname{dim} \mathfrak{w} \in\{0, \ldots, n-1\}$. Let $S_{V, \mathfrak{w}}$ be the connected subgroup of $S U(1, n)$ with Lie algebra $\mathfrak{s}_{V, \mathfrak{w}}$. We will show that the action of $S_{V, \mathfrak{w}}$ induces a homogeneous polar foliation of $\mathbb{C} H^{n}$ with cohomogeneity equal to $\operatorname{dim} \mathfrak{w}$ if $V=\mathfrak{a}$ and equal to $\operatorname{dim} \mathfrak{w}+1$ if $V=\{0\}$. Moreover, the actions of two such subgroups $S_{V, \mathfrak{w}}$ and $S_{V^{\prime}, \mathfrak{w}^{\prime}}$ are orbit equivalent if and only if $\operatorname{dim} V=\operatorname{dim} V^{\prime}$ and $\operatorname{dim} \mathfrak{w}=\operatorname{dim} \mathfrak{w}^{\prime}$. We therefore can define $S_{a, b}:=S_{V, \mathfrak{w}}$ with $a=\operatorname{dim} V \in\{0,1\}$ and $b=\operatorname{dim} \mathfrak{w} \in\{0, \ldots, n-1\}$, and up to isometric congruence $S_{a, b}$ is well defined. The group $S_{1,0}$ acts transitively on $\mathbb{C} H^{n}$, and the group $S_{0,0}$ induces a foliation of $\mathbb{C} H^{n}$ by horospheres. Our main result states:

Main Theorem. Every nontrivial homogeneous polar foliation of $\mathbb{C} H^{n}$, $n \geq 2$, is up to isometric congruence one of the following:

(i) The homogeneous polar foliation induced by $S_{0, b}, b \in\{0, \ldots, n-1\}-$ in this case the codimension of the foliation is equal to $b+1$ and all leaves are contained in horospheres of $\mathbb{C} H^{n}$; 
(ii) The homogeneous polar foliation induced by $S_{1, b}, b \in\{1, \ldots, n-1\}-$ in this case the codimension of the foliation is equal to $b$ and no leaf is contained in a horosphere of $\mathbb{C} H^{n}$.

It is worthwhile to point out that none of the foliations in (i) and (ii) has a totally geodesic leaf.

This paper is organized as follows. In Section 2, we present some relevant material about the structure theory of the Lie algebra of $S U(1, n)$, and in Section 3 we prove that the action of $S_{V, \mathfrak{w}}$ induces a homogeneous polar foliation of $\mathbb{C} H^{n}$. In Section 4, we present the proof of the Main Theorem.

\section{Preliminaries}

For the concepts and notation on Lie groups and Lie algebras we follow [8]. For more information on the complex hyperbolic space and its relation to Damek-Ricci spaces see [4].

We denote by $\mathbb{C} H^{n}, n \geq 2$, the complex hyperbolic space equipped with the Bergman metric normalized so that the holomorphic sectional curvature is equal to -1 . It can be realized as the Riemannian symmetric space $G / K$ with $G=S U(1, n)$ and $K=S(U(1) U(n))$ and a $G$-invariant Riemannian metric induced by the Killing form of the Lie algebra $\mathfrak{g}$ of $G$. Here, $G$ is the identity component of the isometry group of $\mathbb{C} H^{n}$ and $K$ is the isotropy subgroup of $G$ at a point $o \in \mathbb{C} H^{n}$, which we will fix from now on. Let $B$ denote the Killing form of $\mathfrak{g}=\mathfrak{s u}(1, n)$, the Lie algebra of $S U(1, n)$. If $\mathfrak{p}$ is the orthogonal complement of $\mathfrak{k}$ in $\mathfrak{g}$ with respect to $B$, then we have a Cartan decomposition $\mathfrak{g}=\mathfrak{k} \oplus \mathfrak{p}$. The Killing form is negative definite on $\mathfrak{k}$ and positive definite on $\mathfrak{p}$. Let $\theta$ be the Cartan involution of $\mathfrak{g}$ with respect to this Cartan decomposition, i.e., $\theta$ acts as the identity on $\mathfrak{k}$ and as minus the identity on $\mathfrak{p}$. Then we can define a positive definite inner product, the so-called Killing metric, on $\mathfrak{g}$ by $\langle X, Y\rangle=-B(\theta X, Y)$ for all $X, Y \in \mathfrak{g}$. Moreover, we have $\langle\operatorname{ad}(X) Y, Z\rangle=-\langle Y, \operatorname{ad}(\theta X) Z\rangle$ for all $X, Y, Z \in \mathfrak{g}$. As usual, ad denotes the adjoint map at Lie algebra level, $\operatorname{ad}(X) Y=[X, Y]$, whereas Ad will denote the adjoint map at Lie group level. Recall that $\mathfrak{p}$ can be identified with the tangent space $T_{o} \mathbb{C} H^{n}$ and hence can be viewed naturally as a complex vector space.

We choose a maximal abelian subspace $\mathfrak{a}$ of $\mathfrak{p}$. Then $\mathfrak{a}$ is one-dimensional. This abelian subspace induces a restricted root space decomposition $\mathfrak{g}=$ $\mathfrak{g}_{-2 \alpha} \oplus \mathfrak{g}_{-\alpha} \oplus \mathfrak{g}_{0} \oplus \mathfrak{g}_{\alpha} \oplus \mathfrak{g}_{2 \alpha}$, where $\mathfrak{g}_{\lambda}=\{X \in \mathfrak{g}: \operatorname{ad}(H) X=\lambda(H) X$ for all $H \in \mathfrak{a}\}$ and $\lambda \in \mathfrak{a}^{*}$. Recall that $\left[\mathfrak{g}_{\lambda}, \mathfrak{g}_{\mu}\right]=\mathfrak{g}_{\lambda+\mu}$. The set $\Sigma=\{-2 \alpha,-\alpha, \alpha, 2 \alpha\}$ 
is called the set of roots. It is known that $\theta \mathfrak{g}_{\lambda}=\mathfrak{g}_{-\lambda}$ for any $\lambda \in \Sigma \cup\{0\}$, and $\mathfrak{g}_{0}=\mathfrak{k}_{0} \oplus \mathfrak{a}$, where $\mathfrak{k}_{0}=\mathfrak{g}_{0} \cap \mathfrak{k}$. It can be seen that $\mathfrak{k}_{0}$ is isomorphic to $\mathfrak{u}(n-1)$, and that $\mathfrak{g}_{2 \alpha}$ is one-dimensional. From now on, we introduce an ordering in $\Sigma$ such that $\alpha$ and $2 \alpha$ are positive roots. This choice selects precisely one unit vector $B$ in $\mathfrak{a}$ for which $\alpha(B)=1 / 2$.

Let $\mathfrak{n}=\mathfrak{g}_{\alpha} \oplus \mathfrak{g}_{2 \alpha}$, which is a nilpotent subalgebra of $\mathfrak{g}$ isomorphic to the $(2 n-1)$-dimensional Heisenberg algebra. Then $\mathfrak{g}=\mathfrak{k} \oplus \mathfrak{a} \oplus \mathfrak{n}$ is an Iwasawa decomposition of $\mathfrak{g}$. It is known that the connected subgroup $A N$ of $G$ whose Lie algebra is $\mathfrak{a} \oplus \mathfrak{n}$ acts simply transitively on $\mathbb{C} H^{n}$. We endow $A N$, and hence $\mathfrak{a} \oplus \mathfrak{n}$, with the left-invariant metric $\langle\cdot, \cdot\rangle_{A N}$ and the complex structure $J$ that make $\mathbb{C} H^{n}$ and $A N$ isometric. If $X, Y \in \mathfrak{a} \oplus \mathfrak{n} \cong T_{1} A N$ are considered as left-invariant vector fields then the relation between the Killing form and the inner product on $\mathfrak{a} \oplus \mathfrak{n}$ is given by $\langle X, Y\rangle_{A N}=\left\langle X_{\mathfrak{a}}, Y_{\mathfrak{a}}\right\rangle+$ $\frac{1}{2}\left\langle X_{\mathfrak{n}}, Y_{\mathfrak{n}}\right\rangle$, where subscript means orthogonal projection. The complex structure $J$ on $\mathfrak{a} \oplus \mathfrak{n}$ is such that $\mathfrak{g}_{\alpha}$ is $J$-invariant, thus $\mathfrak{g}_{\alpha}$ is a complex vector subspace, and $J \mathfrak{a}=\mathfrak{g}_{2 \alpha}$. Therefore, we can define $Z=J B \in \mathfrak{g}_{2 \alpha}$. Notice that, while $B$ and $Z$ are unit vectors with respect to the $\mathfrak{a} \oplus \mathfrak{n}$ metric, we have $\langle B, B\rangle=1$ but $\langle Z, Z\rangle=2$ with respect to the Killing metric. With this notation, the Lie bracket in $\mathfrak{a} \oplus \mathfrak{n}$ can be written as

$$
[a B+U+x Z, b B+V+y Z]=-\frac{b}{2} U+\frac{a}{2} V+\left(-b x+a y+\frac{1}{2}\langle J U, V\rangle\right) Z
$$

where $a, b, x, y \in \mathbb{R}$, and $U, V \in \mathfrak{g}_{\alpha}$.

We can also define $\mathfrak{p}_{\lambda}=(1-\theta) \mathfrak{g}_{\lambda} \subset \mathfrak{p}$ and $\mathfrak{k}_{\lambda}=(1+\theta) \mathfrak{g}_{\lambda} \subset \mathfrak{k}$. Then we have $\mathfrak{p}=\mathfrak{a} \oplus \mathfrak{p}_{\alpha} \oplus \mathfrak{p}_{2 \alpha}, \mathfrak{p}_{\alpha}$ is complex, and $\mathfrak{p}_{2 \alpha}$ is one-dimensional. If $i$ denotes the complex structure of $\mathfrak{p}$ then we have

$$
\mathrm{i} B=\frac{1}{2}(1-\theta) Z \quad \text { and } \quad \mathrm{i}(1-\theta) U=(1-\theta) J U
$$

There are exactly two conjugacy classes of Cartan subalgebras in $\mathfrak{g}$. We are interested in the so-called maximally noncompact ones. These are, up to conjugacy, of the form $\mathfrak{t} \oplus \mathfrak{a}$, where $\mathfrak{t}$ is some abelian subspace of $\mathfrak{k}_{0}$. Then, $[\mathfrak{t}, \mathfrak{a}]=\left[\mathfrak{t}, \mathfrak{g}_{2 \alpha}\right]=0$, and $\left[\mathfrak{t}, \mathfrak{g}_{\alpha}\right] \subset \mathfrak{g}_{\alpha}$. The vector space $\mathfrak{t} \oplus \mathfrak{a} \oplus \mathfrak{n}$ turns out to be a maximal solvable subalgebra of $\mathfrak{g}$, of the so-called maximal noncompact type.

To finish this section, we prove the following result, which will be used several times henceforth. 
Lemma 2.1. We have

$$
\begin{aligned}
& {[\theta U, Z]=-J U \text { for all } U \in \mathfrak{g}_{\alpha} ;} \\
& \langle T,(1+\theta)[\theta U, V]\rangle=2\langle[T, U], V\rangle \text { for all } T \in \mathfrak{t} \text { and } U, V \in \mathfrak{g}_{\alpha} .
\end{aligned}
$$

Proof. Assume that $U \in \mathfrak{g}_{\alpha}$. It is clear that $[\theta U, Z] \in \mathfrak{g}_{\alpha}$. Moreover, if $V$ is another element in $\mathfrak{g}_{\alpha}$ we have $\langle[\theta U, Z], V\rangle=-\langle Z,[U, V]\rangle=$ $-\frac{1}{2}\langle J U, V\rangle\langle Z, Z\rangle=-\langle J U, V\rangle$, where we have used $\langle Z, Z\rangle=2$. This implies (2.2).

Now let $T \in \mathfrak{t}$ and $U, V \in \mathfrak{g}_{\alpha}$. Then

$$
\begin{aligned}
\langle T,(1+\theta)[\theta U, V]\rangle & =\langle T,[\theta U, V]\rangle+\langle T,[U, \theta V]\rangle=-\langle[U, T], V\rangle+\langle[V, T], U\rangle \\
& =2\langle[T, U], V\rangle,
\end{aligned}
$$

from where (2.3) follows.

\section{Polar foliations}

The following criterion for homogeneous polar foliations was proved by Gorodski in [7] for Riemannian symmetric spaces of compact type, but the proof for the noncompact type is analogous (see also [1]).

Theorem 3.1. Let $M=G / K$ be a Riemannian symmetric space of noncompact type and $H$ be a connected closed subgroup of $G$ whose orbits form a homogeneous foliation $\mathcal{F}$ of $M$. Consider the corresponding Cartan decomposition $\mathfrak{g}=\mathfrak{k} \oplus \mathfrak{p}$ and define

$$
\mathfrak{h}_{\mathfrak{p}}^{\perp}=\{\xi \in \mathfrak{p}:\langle\xi, Y\rangle=0 \text { for all } Y \in \mathfrak{h}\} .
$$

Then the action of $H$ on $M$ is polar if and only if $\mathfrak{h}_{\mathfrak{p}}^{\perp}$ is a Lie triple system in $\mathfrak{p}$ and $\mathfrak{h}$ is orthogonal to the subalgebra $\left[\mathfrak{h}_{\mathfrak{p}}^{\perp}, \mathfrak{h}_{\mathfrak{p}}^{\perp} \oplus \mathfrak{h}_{\mathfrak{p}}^{\perp}\right.$ of $\mathfrak{g}$. In this case, let $H_{\mathfrak{p}}^{\perp}$ be the connected subgroup of $G$ with Lie algebra $\left[\mathfrak{h}_{\mathfrak{p}}^{\perp}, \mathfrak{h}_{\mathfrak{p}}^{\perp}\right] \oplus \mathfrak{h}_{\mathfrak{p}}^{\perp}$. Then the orbit $\mathcal{S}=H_{\mathfrak{p}}^{\perp}$ o is a section of the $H$-action on $M$.

As a first application of this theorem we construct examples of homogeneous polar foliations of the complex hyperbolic space $\mathbb{C} H^{n}$. Here and henceforth the symbol $\ominus$ denotes orthogonal complement.

Theorem 3.2. Let $\mathfrak{w}$ be a real subspace of $\mathfrak{g}_{\alpha}$ and $V$ be a subspace of $\mathfrak{a}$ (then, either $V=\{0\}$ or $V=\mathfrak{a}$ ). Let $S_{V, \mathfrak{w}}$ be the connected subgroup of $A N$ whose Lie algebra is $\mathfrak{s}_{V, \mathfrak{w}}=V \oplus\left(\mathfrak{g}_{\alpha} \ominus \mathfrak{w}\right) \oplus \mathfrak{g}_{2 \alpha}$. Then $S_{V, \mathfrak{w}}$ induces a homogeneous polar foliation of $\mathbb{C} H^{n}$. 
Proof. It is clear that $\mathfrak{s}_{V, \mathfrak{w}}$ is a subalgebra of $\mathfrak{a} \oplus \mathfrak{n}$. Since $S_{V, \mathfrak{w}}$ is contained in $A N$ it is obvious that it induces a homogeneous foliation of $\mathbb{C} H^{n}$. So, it remains to use Theorem 3.1 to show that it also acts polarly on $\mathbb{C} H^{n}$.

It is easy to calculate that

$$
\left(\mathfrak{s}_{V, \mathfrak{w}}\right)_{\mathfrak{p}}^{\perp}=(\mathfrak{a} \ominus V) \oplus(1-\theta) \mathfrak{w} .
$$

The subspace above is real in $\mathfrak{p}$ (use for example (2.1)), and hence it is a Lie triple system of $\mathfrak{p}$. Now, if $W, \tilde{W} \in \mathfrak{w}$, we have $[(1-\theta) W,(1-\theta) \tilde{W}]=$ $(1+\theta)[W, \tilde{W}]-(1+\theta)[\theta W, \tilde{W}]=-(1+\theta)[\theta W, \tilde{W}] \in \mathfrak{k}_{0}$, which is orthogonal to $\mathfrak{a} \oplus \mathfrak{n}$ and thus to $\mathfrak{s}_{V, \mathfrak{w}}$. Also, $[B,(1-\theta) W]=\frac{1}{2}(1+\theta) W$, and since $\mathfrak{w}$ is orthogonal to $\mathfrak{s}_{V, \mathfrak{w}}$, so is the latter element of $\mathfrak{k}_{\alpha}$. This finishes the proof of the theorem.

Denote by $K_{0}$ the subgroup of $K$ with Lie algebra $\mathfrak{k}_{0}$. The adjoint action of $K_{0}$ on $\mathfrak{g}_{\alpha}$ is isomorphic to the standard action of $U(n-1)$ on $\mathbb{C}^{n-1}$. This implies that for any two real subspaces $\mathfrak{w}$ and $\mathfrak{w}^{\prime}$ of $\mathfrak{g}_{\alpha}$ with dim $\mathfrak{w}=$ $\operatorname{dim} \mathfrak{w}^{\prime}$ the subgroups $S_{V, \mathfrak{w}}$ and $S_{V, \mathfrak{w}^{\prime}}$ are conjugate in $S U(1, n)$. Hence the corresponding two homogeneous polar foliations on $\mathbb{C} H^{n}$ are isometrically congruent to each other.

We will now proceed with the proof of the Main Theorem.

\section{Proof of the main theorem}

Assume that $H$ is a connected closed subgroup of $G=S U(1, n)$ acting polarly on $\mathbb{C} H^{n}$ in such a way that the orbits of $H$ form a homogeneous foliation of $\mathbb{C} H^{n}$. We denote by $\mathfrak{h}$ the Lie algebra of $H$. According to [1] we have:

Theorem 4.1. Let $M$ be a Hadamard manifold and let $H$ be a connected closed subgroup of $I(M)$ whose orbits form a homogeneous foliation $\mathcal{F}$ of $M$. Then there exists a connected closed solvable subgroup $S$ of $H$ such that the leaves of $\mathcal{F}$ coincide with the orbits of $S$. Moreover, all the orbits of $S$ or of $H$ are principal.

Theorem 4.1 implies that we may assume that $\mathfrak{h}$ is solvable. Thus $\mathfrak{h}$ is contained in a maximal solvable subalgebra of $\mathfrak{g}$. We can give a more precise statement in our situation.

Proposition 4.1. Let $M$ be a symmetric space of noncompact type and rank one. Assume that $H$ acts polarly on $M$ inducing a foliation. Then the 
action of $H$ is orbit equivalent to the action of a Lie group whose Lie algebra is contained in a maximally noncompact Borel subalgebra.

Proof. We write $M=G / K$, where $G$ is the connected component of the isometry group of $M$. By Theorem 4.1 we may assume that $H$ is solvable and closed in $G$.

Let $\mathfrak{b}$ be a maximal solvable subalgebra of $\mathfrak{g}$ containing $\mathfrak{h}$. Then there exists a Cartan decomposition $\mathfrak{g}=\mathfrak{k} \oplus \mathfrak{p}$ such that $\mathfrak{b}=\mathfrak{t} \oplus \tilde{\mathfrak{a}} \oplus \tilde{\mathfrak{n}}$, with $\mathfrak{t} \subset \mathfrak{k}$, $\tilde{\mathfrak{a}} \subset \mathfrak{p}$, and such that $\mathfrak{t} \oplus \tilde{\mathfrak{a}}$ is Cartan subalgebra of $\mathfrak{g}[12]$. Here, $\tilde{\mathfrak{n}}$ is defined as $\tilde{\mathfrak{n}}=\oplus_{\tilde{\lambda} \in \tilde{\Sigma}^{+}} \tilde{\mathfrak{g}}_{\tilde{\lambda}}$, where $\tilde{\Sigma}$ is the set of roots with respect to $\tilde{\mathfrak{a}}, \tilde{\mathfrak{g}}_{\tilde{\lambda}}=\{X \in \mathfrak{g}$ : $\operatorname{ad}(H) X=\tilde{\lambda}(H) X$ for all $H \in \tilde{\mathfrak{a}}\}$, and $\tilde{\Sigma}^{+}$is the set of positive roots with respect to some choice of ordering. Since $M$ has rank one, either $\tilde{\mathfrak{a}}=\{0\}$ or $\operatorname{dim} \tilde{\mathfrak{a}}=1$. If $\tilde{\mathfrak{a}}=\{0\}$ then $\mathfrak{b}=\mathfrak{t}$ is compact. Hence, the action of $H$ is orbit equivalent to the action of a compact subgroup, and thus has a fixed point by Cartan's fixed point theorem. Since $H$ induces a foliation, it follows that the orbits of $H$ are points. Therefore, we may assume $\operatorname{dim} \tilde{\mathfrak{a}}=1$, and thus $\mathfrak{b}$ is maximally noncompact. We conclude that $\mathfrak{h}$ is a subalgebra of $\mathfrak{t} \oplus \mathfrak{a} \oplus \mathfrak{n}$, where $\mathfrak{a} \subset \mathfrak{p}$ is a maximal abelian subspace.

In view of Proposition 4.1 we may assume that $\mathfrak{h}$ is a subalgebra of $\mathfrak{t} \oplus$ $\mathfrak{a} \oplus \mathfrak{n}$, where $\mathfrak{a} \subset \mathfrak{p}$ is a maximal abelian subspace and $\mathfrak{t} \oplus \mathfrak{a}$ is a maximally noncompact Cartan subalgebra of $\mathfrak{g}$.

Let $\mathcal{S}$ be a section of the action of $H$. Then, according to Theorem 3.1, $\mathcal{S}=H_{\mathfrak{p}}^{\perp} \cdot o$, where $H_{\mathfrak{p}}^{\perp}$ is the connected subgroup of $G=S U(1, n)$ whose Lie algebra is $\left[\mathfrak{h}_{\mathfrak{p}}^{\perp}, \mathfrak{h}_{\mathfrak{p}}^{\perp}\right] \oplus \mathfrak{h}_{\mathfrak{p}}^{\perp} \subset \mathfrak{k} \oplus \mathfrak{p}=\mathfrak{g}$, where $\mathfrak{h}_{\mathfrak{p}}^{\perp}=\{\xi \in \mathfrak{p}:\langle\xi, Y\rangle=0$ for all $Y \in \mathfrak{h}\} \subset \mathfrak{p}$. Since $\mathfrak{h}_{\mathfrak{p}}^{\perp}$ is a Lie triple system it follows that $\mathfrak{h}_{\mathfrak{p}}^{\perp}$ is either real or complex, and thus $\mathcal{S}$ is also either real or complex. We deal first with the complex case.

Proposition 4.2. If the section $\mathcal{S}$ of $H$ is complex, then either $H$ acts transitively on $\mathbb{C} H^{n}$ or the orbits of $H$ are points.

Proof. Let $\pi: \mathfrak{h}_{\mathfrak{p}}^{\perp} \rightarrow \mathfrak{a} \oplus \mathfrak{p}_{2 \alpha}$ be the orthogonal projection of $\mathfrak{h}_{\mathfrak{p}}^{\perp}$ onto $\mathfrak{a} \oplus \mathfrak{p}_{2 \alpha}$. Note that $\pi$ is $J$-linear, and thus $\pi\left(\mathfrak{h}_{\mathfrak{p}}^{\perp}\right)$ is either $\{0\}$ or $\mathfrak{a} \oplus \mathfrak{p}_{2 \alpha}$.

Assume first that $\pi\left(\mathfrak{h}_{\mathfrak{p}}^{\perp}\right)=\{0\}$. Then it is clear that $\mathfrak{h}_{\mathfrak{p}}^{\perp}$ is a complex subspace of $\mathfrak{p}_{\alpha}$. Since $B$ and $Z$ are orthogonal to $\mathfrak{h}_{\mathfrak{p}}^{\perp}$, there exist $S, T \in \mathfrak{t}$ such that $S+B, T+Z \in \mathfrak{h}$. Then, $Z=[S+B, T+Z] \in \mathfrak{h}$. Let $V \in \mathfrak{g}_{\alpha}$ such that $(1-\theta) V \in \mathfrak{h}_{\mathfrak{p}}^{\perp}$. Theorem 3.1 implies

$$
0=\langle Z,[(1-\theta) V,(1-\theta) J V]\rangle=\langle Z,[V, J V]\rangle=\frac{1}{2}\langle V, V\rangle\langle Z, Z\rangle=\|V\|^{2},
$$


and so $\mathfrak{h}_{\mathfrak{p}}^{\perp}=\{0\}$. Hence the section is zero-dimensional and therefore the action of $H$ must be transitive.

Now assume that $\pi\left(\mathfrak{h}_{\mathfrak{p}}^{\perp}\right)=\mathfrak{a} \oplus \mathfrak{p}_{2 \alpha}$. As $\pi$ is $J$-linear, ker $\pi$ is a complex subspace of $\mathfrak{p}_{\alpha}$. Then $\mathfrak{h}_{\mathfrak{p}}^{\perp} \ominus \operatorname{ker} \pi$, the orthogonal complement of ker $\pi$ in $\mathfrak{h}_{\mathfrak{p}}^{\perp}$, has complex dimension one, so there is a unique vector $Y \in \mathfrak{h}_{\mathfrak{p}}^{\perp} \ominus \operatorname{ker} \pi$ such that $\pi(Y)=B$. Thus, we can write $Y=B+(1-\theta) X$, with $X \in \mathfrak{g}_{\alpha}$ and $X$ (or $(1-\theta) X)$ orthogonal to ker $\pi$. In view of $(2.1)$, we can also write

$$
\begin{aligned}
\mathfrak{h}_{\mathfrak{p}}^{\perp} & =\mathbb{C}(B+(1-\theta) X) \oplus \operatorname{ker} \pi \\
& =\mathbb{R}(B+(1-\theta) X) \oplus \mathbb{R}\left((1-\theta) J X+\frac{1}{2}(1-\theta) Z\right) \oplus \operatorname{ker} \pi
\end{aligned}
$$

It is obvious that $\|X\|^{2} B-X$, and $-J X+\|X\|^{2} Z$ are orthogonal to $\mathfrak{h}_{\mathfrak{p}}^{\perp}$ (recall that $\|B\|^{2}=1$, and $\|Z\|^{2}=2$, where $\|\cdot\|$ is the norm induced by $\langle\cdot, \cdot\rangle)$. Hence, there exist $S, T \in \mathfrak{t}$ such that $S+\|X\|^{2} B-X, T-J X+$ $\|X\|^{2} Z \in \mathfrak{h}$.

Using (2.2) we obtain

$$
\begin{aligned}
{\left[\mathfrak{h}_{\mathfrak{p}}^{\perp}, \mathfrak{h}_{\mathfrak{p}}^{\perp}\right] \ni } & {\left[B+(1-\theta) X,(1-\theta) J X+\frac{1}{2}(1-\theta) Z\right] } \\
= & \frac{1}{2}(1+\theta) J X+\frac{1}{2}(1+\theta) Z+(1+\theta)[X, J X] \\
& -(1+\theta)[\theta X, J X]-\frac{1}{2}(1+\theta)[\theta X, Z] \\
= & -(1+\theta)[\theta X, J X]+(1+\theta) J X+\frac{1}{2}\left(1+\|X\|^{2}\right)(1+\theta) Z
\end{aligned}
$$

Taking inner product of the previous vector with $T-J X+\|X\|^{2} Z \in \mathfrak{h}$, and using the fact that $\operatorname{ad}(T)$ is skew-symmetric, and (2.3), we obtain by Theorem 3.1

$$
\begin{aligned}
0 & =-\langle(1+\theta)[\theta X, J X], T\rangle-\|X\|^{2}+\frac{\|X\|^{2}}{2}\left(1+\|X\|^{2}\right)\|Z\|^{2} \\
& =-2\langle[T, X], J X\rangle+\|X\|^{4} .
\end{aligned}
$$

Therefore, we obtain $\langle[T, X], J X\rangle=\|X\|^{4} / 2$.

On the other hand, since $\mathfrak{h}$ is a Lie algebra, and $\left[\mathfrak{t}, \mathfrak{g}_{2 \alpha}\right]=0$, we have that

$$
\begin{aligned}
\mathfrak{h} \ni[S & \left.+\|X\|^{2} B-X, T-J X+\|X\|^{2} Z\right] \\
& =[T, X]-[S, J X]-\frac{\|X\|^{2}}{2} J X+\|X\|^{2}\left(\|X\|^{2}+\frac{1}{2}\right) Z .
\end{aligned}
$$


Taking inner product with $(1-\theta) J X+\frac{1}{2}(1-\theta) Z \in \mathfrak{h}_{\mathfrak{p}}^{\perp}$, and using the fact that $\operatorname{ad}(S)$ is skew-symmetric, yields

$$
0=\langle[T, X], J X\rangle-\frac{\|X\|^{4}}{2}+\|X\|^{2}\left(\|X\|^{2}+\frac{1}{2}\right)=\|X\|^{2}\left(\|X\|^{2}+\frac{1}{2}\right) .
$$

Therefore $X=0$, and $\mathfrak{h}_{\mathfrak{p}}^{\perp}=\mathfrak{a} \oplus \mathfrak{p}_{2 \alpha} \oplus \operatorname{ker} \pi$. Since ker $\pi$ is complex we can find $S, T \in \mathfrak{t}$, and $V \in \mathfrak{g}_{\alpha}$, such that $S+V, T+J V \in \mathfrak{h}$. Then

$$
\begin{aligned}
0 & =\langle[S+V, T+J V],(1-\theta) Z\rangle \\
& =\left\langle[S, J V]-[T, V]+\frac{1}{2}\|V\|^{2} Z,(1-\theta) Z\right\rangle=\|V\|^{2} .
\end{aligned}
$$

This implies $\operatorname{ker} \pi=\mathfrak{p}_{\alpha}$ and $\mathfrak{h} \subset \mathfrak{t}$. Therefore $H$ is compact. By Cartan's fixed point theorem $H$ has a fixed point and thus fixes $\mathbb{C} H^{n}$ pointwise.

Altogether this proves that a homogeneous polar foliation of the complex hyperbolic space cannot have a nontrivial complex section.

From now on we assume that the section is real, that is, $\mathfrak{h}_{\mathfrak{p}}^{\perp}$ is a real subspace of $\mathfrak{p}$. Consider again the orthogonal projection $\pi: \mathfrak{h}_{\mathfrak{p}}^{\perp} \rightarrow \mathfrak{a} \oplus \mathfrak{p}_{2 \alpha}$. Two different possibilities arise.

Case $1 \operatorname{dim} \pi\left(\mathfrak{h}_{\mathfrak{p}}^{\perp}\right)=2$ : We will show that this possibility cannot hold. We start with an algebraic result.

Lemma 4.1. The subspace $\mathfrak{h}_{\mathfrak{p}}^{\perp}$ can be written as

$$
\mathfrak{h}_{\mathfrak{p}}^{\perp}=\mathbb{R}(B+(1-\theta) X) \oplus \mathbb{R}((1-\theta) Y+(1-\theta) Z) \oplus(1-\theta) \mathfrak{w}
$$

where $X, Y \in \mathfrak{g}_{\alpha}, \mathfrak{w}$ is a real subspace of $\mathfrak{g}_{\alpha}, \mathbb{C} X$ and $\mathbb{C} Y$ are perpendicular to $\mathfrak{w}$, and $\langle X, J Y\rangle=1$.

Proof. Since ker $\pi \subset \mathfrak{p}_{\alpha}$, we can find a subspace $\mathfrak{w} \subset \mathfrak{g}_{\alpha}$ such that $\operatorname{ker} \pi=$ $(1-\theta) \mathfrak{w}$. By hypothesis, there exists a unique $\xi \in \mathfrak{h}_{\mathfrak{p}}^{\perp} \ominus \operatorname{ker} \pi$ such that $\pi(\xi)=B$. Hence, there exists $X \in \mathfrak{g}_{\alpha}$ such that $\xi=B+(1-\theta) X \in \mathfrak{h}_{\mathfrak{p}}^{\perp}$. Similarly, there exists a unique $\eta \in \mathfrak{h}_{\mathfrak{p}}^{\perp} \ominus \operatorname{ker} \pi$ such that $\pi(\eta)=(1-\theta) Z$, and thus, there exists $Y \in \mathfrak{g}_{\alpha}$ such that $\eta=(1-\theta) Z+(1-\theta) Y \in \mathfrak{h}_{\mathfrak{p}}^{\perp}$. Clearly, $\mathfrak{h}_{\mathfrak{p}}^{\perp}=\mathbb{R} \xi \oplus \mathbb{R} \eta \oplus \operatorname{ker} \pi=\mathbb{R}(B+(1-\theta) X) \oplus \mathbb{R}((1-\theta) Y+(1-\theta) Z)$ $\oplus(1-\theta) \mathfrak{w}$.

Since $\mathfrak{h}_{\mathfrak{p}}^{\perp}$ is real, using $(2.1)$ we obtain $0=\langle\xi, i \eta\rangle=-2+2\langle X, J Y\rangle$. Let $W \in \mathfrak{w} \subset \mathfrak{g}_{\alpha}$ be arbitrary. By definition of $\xi$, we obtain $0=\langle(1-\theta) W, \xi\rangle=$ 
$2\langle W, X\rangle$, and since $\mathfrak{h}_{\mathfrak{p}}^{\perp}$ is real, $0=\langle(1-\theta) W, \mathrm{i} \xi\rangle=2\langle W, J X\rangle$. This implies that $\mathbb{C} X$ is orthogonal to $\mathfrak{w}$. We can prove in a similar way that $\mathbb{C} Y$ is orthogonal to $\mathfrak{w}$, and hence the result follows.

In view of Lemma 4.1 our first observation is that

for each $U \in \mathfrak{g}_{\alpha} \ominus \mathfrak{w}, \quad-\langle U, X\rangle B+U-\frac{1}{2}\langle U, Y\rangle Z$ is orthogonal to $\mathfrak{h}_{\mathfrak{p}}^{\perp}$.

Hence, for each $U \in \mathfrak{g}_{\alpha} \ominus \mathfrak{w}$ there exists $T_{U} \in \mathfrak{t}$ such that $T_{U}-\langle U, X\rangle B+$ $U-\frac{1}{2}\langle U, Y\rangle Z \in \mathfrak{h}$.

Using (2.2) we also obtain

$$
\begin{aligned}
{[B} & +(1-\theta) X,(1-\theta) Y+(1-\theta) Z] \\
& =-(1+\theta)[\theta X, Y]+\frac{1}{2}(1+\theta) Y+(1+\theta) J X+\frac{1}{2}(1+\theta) Z .
\end{aligned}
$$

Lemma 4.2. We have that $Y \in \mathbb{C} X$. More explicitly, there exists $\gamma \in \mathbb{R}$ such that

$$
Y=\gamma X-\frac{1}{\|X\|^{2}} J X
$$

Proof. According to (4.1), there exist $T_{X}, T_{Y} \in \mathfrak{t}$ such that

$$
T_{X}-\|X\|^{2} B+X-\frac{1}{2}\langle X, Y\rangle Z, \quad T_{Y}-\langle X, Y\rangle B+Y-\frac{\|Y\|^{2}}{2} Z \in \mathfrak{h} .
$$

Since $\mathfrak{h}$ is orthogonal to $\left[\mathfrak{h}_{\mathfrak{p}}^{\perp}, \mathfrak{h}_{\mathfrak{p}}^{\perp}\right]$ by Theorem 3.1, taking inner product of $T_{Y}-\langle X, Y\rangle B+Y-\frac{1}{2}\|Y\|^{2} Z$ with (4.2), and using (2.3) we obtain

$$
0=-\left\langle T_{Y},(1+\theta)[\theta X, Y]\right\rangle+\langle J X, Y\rangle=-2\left\langle\left[T_{Y}, X\right], Y\right\rangle-1
$$

so $\left\langle\left[T_{Y}, Y\right], X\right\rangle=1 / 2$.

Now, since $\mathfrak{h}$ is a Lie algebra

$$
\begin{aligned}
\mathfrak{h} \ni & {\left[T_{X}-\|X\|^{2} B+X-\frac{1}{2}\langle X, Y\rangle Z, T_{Y}-\langle X, Y\rangle B+Y-\frac{\|Y\|^{2}}{2} Z\right] } \\
= & {\left[T_{X}, Y\right]-\left[T_{Y}, X\right]+\frac{1}{2}\left(\langle X, Y\rangle X-\|X\|^{2} Y\right) } \\
& +\left(\frac{\|X\|^{2}\|Y\|^{2}}{2}-\frac{\langle X, Y\rangle^{2}}{2}-\frac{1}{2}\right) Z .
\end{aligned}
$$


Taking inner product with $(1-\theta) Y+(1-\theta) Z \in \mathfrak{h}_{\mathfrak{p}}^{\perp}$, using the skewsymmetry of $\operatorname{ad}\left(T_{X}\right)$, and $\left\langle\left[T_{Y}, Y\right], X\right\rangle=1 / 2$, we obtain

$$
\begin{aligned}
0 & =\left\langle\left[T_{X}, Y\right]-\left[T_{Y}, X\right], Y\right\rangle+\frac{1}{2}\left(\|X\|^{2}\|Y\|^{2}-\langle X, Y\rangle^{2}\right)+\langle J X, Y\rangle \\
& =\frac{1}{2}\left(\|X\|^{2}\|Y\|^{2}-\langle X, Y\rangle^{2}-1\right) .
\end{aligned}
$$

This implies

$$
\|X\|^{2}\|Y\|^{2}-\langle X, Y\rangle^{2}=1=\langle J X, Y\rangle^{2} .
$$

We may write $Y=\gamma X+\delta J X+E$ with $\gamma, \delta \in \mathbb{R}$ and $E \in \mathfrak{g}_{\alpha} \ominus \mathbb{C} X$. Then, the equation above reads $\|X\|^{2}\|E\|^{2}=0$, and since $X \neq 0$ we obtain $E=0$. As $\langle X, J Y\rangle=1$, this readily implies the result.

Let $g \in G$ be an arbitrary isometry. The groups $H$ and $I_{g}(H)=g H g^{-1}$ are conjugate, and since $H$ induces a foliation so does $I_{g}(H)$. Since all the orbits of a homogeneous foliation are principal and $H$ and $I_{g}(H)$ are conjugate, so are their isotropy groups at the origin, $H_{o}$ and $I_{g}(H)_{o}$ respectively. In particular, their Lie algebras have the same dimension, that is, $\operatorname{dim}(\mathfrak{h} \cap \mathfrak{k})=\operatorname{dim}(\operatorname{Ad}(g)(\mathfrak{h}) \cap \mathfrak{k})$. From now on let us choose

$$
g=\exp \left(-\frac{2}{\|X\|^{2}} X\right) .
$$

We will see that $\operatorname{dim}(\mathfrak{h} \cap \mathfrak{k})<\operatorname{dim}(\operatorname{Ad}(g)(\mathfrak{h}) \cap \mathfrak{k})$, thus leading to a contradiction.

Note that the slice representation is trivial at each point because all the orbits are principal. Since $\mathfrak{h} \cap \mathfrak{t}=\mathfrak{h} \cap \mathfrak{k}$ is the isotropy subalgebra of $\mathfrak{h}$ at $o$ and $\mathfrak{h}_{\mathfrak{p}}^{\perp}=\nu_{o}(H \cdot o)$, we therefore obtain $\left[\mathfrak{h} \cap \mathfrak{t}, \mathfrak{h}_{\mathfrak{p}}^{\perp}\right]=0$. Since $\left[\mathfrak{t}, \mathfrak{g}_{\alpha}\right] \subset \mathfrak{g}_{\alpha}$ and $[\mathfrak{t}, \mathfrak{a}]=0$, we obtain from the previous equality that $[\mathfrak{h} \cap \mathfrak{t}, B+(1-\theta) X]=$ 0 , and thus

$$
[\mathfrak{h} \cap \mathfrak{t}, X]=0 \quad \text { and } \quad \operatorname{Ad}(g)(\mathfrak{h} \cap \mathfrak{t})=\mathfrak{h} \cap \mathfrak{t} .
$$

It follows that $\mathfrak{h} \cap \mathfrak{t}=\operatorname{Ad}(g)(\mathfrak{h} \cap \mathfrak{t}) \subset \operatorname{Ad}(g)(\mathfrak{h}) \cap \mathfrak{k}$, and therefore we obtain $\quad \operatorname{dim}(\mathfrak{h} \cap \mathfrak{k})=\operatorname{dim}(\mathfrak{h} \cap \mathfrak{t}) \leq \operatorname{dim}(\operatorname{Ad}(g)(\mathfrak{h}) \cap \mathfrak{t}) \leq \operatorname{dim}(\operatorname{Ad}(g)(\mathfrak{h}) \cap \mathfrak{k})$. From (4.1), there exists $T_{J X} \in \mathfrak{t}$ such that $T_{J X}+J X+\frac{1}{2} Z \in \mathfrak{h}$. We will show the following two facts:

$$
\operatorname{Ad}(g)\left(T_{J X}+J X+\frac{1}{2} Z\right)=T_{J X} \in \operatorname{Ad}(g)(\mathfrak{h}) \cap \mathfrak{t}, \quad \text { and } \quad T_{J X} \notin \mathfrak{h} \cap \mathfrak{t} .
$$

This, and the previous inequalities, exhibit the fact that $\operatorname{dim}(\mathfrak{h} \cap \mathfrak{k})<$ $\operatorname{dim}(\operatorname{Ad}(g)(\mathfrak{h}) \cap \mathfrak{k})$ which gives the desired contradiction. 
We have by (2.3) and (4.2)

$$
\begin{aligned}
0 & =\left\langle T_{J X}+J X+\frac{1}{2} Z,[B+(1-\theta) X,(1-\theta) Y+(1-\theta) Z]\right\rangle \\
& =-2\left\langle\left[T_{J X}, X\right], Y\right\rangle+\|X\|^{2}=\frac{2}{\|X\|^{2}}\left\langle\left[T_{J X}, X\right], J X\right\rangle+\|X\|^{2},
\end{aligned}
$$

and hence,

$$
\left\langle\left[T_{J X}, X\right], J X\right\rangle=-\frac{\|X\|^{4}}{2} .
$$

In particular, since $[\mathfrak{h} \cap \mathfrak{t}, X]=0$ and $X \neq 0$, this implies $T_{J X} \notin \mathfrak{h} \cap \mathfrak{t}$.

Let us then show $\operatorname{Ad}(g)\left(T_{J X}+J X+\frac{1}{2} Z\right)=T_{J X}$. Let $U \in \mathfrak{g}_{\alpha} \ominus$ $(\mathbb{C} X \oplus \mathfrak{w})$ and by $(4.1)$ take $T_{U} \in \mathfrak{t}$ such that $T_{U}+U \in \mathfrak{h}$. Then, by $(2.3)$ and (4.2),

$$
\begin{aligned}
0 & =\left\langle T_{U}+U,[B+(1-\theta) X,(1-\theta) Y+(1-\theta) Z]\right\rangle=-2\left\langle\left[T_{U}, X\right], Y\right\rangle \\
& =\frac{2}{\|X\|^{2}}\left\langle\left[T_{U}, X\right], J X\right\rangle
\end{aligned}
$$

and thus $\left\langle\left[T_{U}, X\right], J X\right\rangle=0$. On the other hand,

$$
\left[T_{J X}, U\right]-\left[T_{U}, J X\right]=\left[T_{J X}+J X+\frac{1}{2} Z, T_{U}+U\right] \in \mathfrak{h},
$$

and hence

$$
\begin{aligned}
0 & =\left\langle\left[T_{J X}, U\right]-\left[T_{U}, J X\right], B+(1-\theta) X\right\rangle=\left\langle\left[T_{J X}, U\right], X\right\rangle-\left\langle\left[T_{U}, J X\right], X\right\rangle \\
& =-\left\langle\left[T_{J X}, X\right], U\right\rangle
\end{aligned}
$$

thus showing $\left[T_{J X}, X\right] \in \mathbb{R} J X \oplus \mathfrak{w}$. Now, for any $W \in \mathfrak{w}$,

$$
\begin{aligned}
0 & =\left\langle T_{J X}+J X+\frac{1}{2} Z,[B+(1-\theta) X,(1-\theta) W]\right\rangle \\
& =\left\langle T_{J X}+J X+\frac{1}{2} Z,-(1+\theta)[\theta X, W]+\frac{1}{2}(1+\theta) W\right\rangle \\
& =-\left\langle T_{J X},(1+\theta)[\theta X, W]\right\rangle=-2\left\langle\left[T_{J X}, X\right], W\right\rangle,
\end{aligned}
$$

using (2.3) once again. This, together with $\left\langle\left[T_{J X}, X\right], J X\right\rangle=-\frac{\|X\|^{4}}{2}$ implies

$$
\left[T_{J X}, X\right]=-\frac{\|X\|^{2}}{2} J X
$$


Now, taking this into account, and $[X, J X]=\frac{\|X\|^{2}}{2} Z$, we obtain

$$
\begin{aligned}
\operatorname{Ad}(g)\left(T_{J X}+J X+\frac{1}{2} Z\right)= & \mathrm{e}^{\operatorname{ad}\left(\frac{-2}{\|X\|^{2}} X\right)}\left(T_{J X}+J X+\frac{1}{2} Z\right) \\
= & T_{J X}+J X+\frac{1}{2} Z-\frac{2}{\|X\|^{2}}\left(\left[X, T_{J X}\right]+[X, J X]\right) \\
& +\frac{2}{\|X\|^{4}}\left[X,\left[X, T_{J X}\right]\right]=T_{J X},
\end{aligned}
$$

which leads to the desired contradiction.

Altogether we have proved

Proposition 4.3. $\operatorname{dim} \pi\left(\mathfrak{h}_{\mathfrak{p}}^{\perp}\right) \leq 1$.

Case $2 \operatorname{dim} \pi\left(\mathfrak{h}_{\mathfrak{p}}^{\perp}\right) \leq 1$ : In this case, there exists a nonzero vector $\xi \in$ $\mathfrak{h}_{\mathfrak{p}}^{\perp} \ominus$ ker $\pi$, which we can write as $\xi=a B+(1-\theta) X+b(1-\theta) Z$, with $X \in$ $\mathfrak{g}_{\alpha}, X$ orthogonal to $\operatorname{ker} \pi$, and $a, b \in \mathbb{R}$. Clearly, $a=b=0$ if $\pi\left(\mathfrak{h}_{\mathfrak{p}}^{\perp}\right)=0$; otherwise, at least one of these numbers is nonzero. Let $\mathfrak{w}$ be a subspace of $\mathfrak{g}_{\alpha}$ such that $\operatorname{ker} \pi=(1-\theta) \mathfrak{w}$. Note that, since $\mathfrak{h}_{\mathfrak{p}}^{\perp}$ is real, $\mathfrak{w}$ is also real, and $0=\langle J \xi,(1-\theta) W\rangle=2\langle J X, W\rangle$ for any $W \in \mathfrak{w}$, so $\mathbb{R} X \oplus \mathfrak{w}$ is a real subspace of $\mathfrak{g}_{\alpha}$.

Lemma 4.3. We have that $\mathfrak{h}_{\mathfrak{p}}^{\perp}=\mathbb{R}(a B+(1-\theta) X) \oplus(1-\theta) \mathfrak{w}$, where $a \in$ $\mathbb{R}, X \in \mathfrak{g}_{\alpha} \ominus \mathfrak{w}$, and $\mathbb{R} X \oplus \mathfrak{w}$ is a real subspace of $\mathfrak{g}_{\alpha}$.

Proof. We only have to prove that $b=0$. Assume, on the contrary, that $b \neq 0$. It is easy to see that $\|X\|^{2} B-a X$ and $-2 b X+\|X\|^{2} Z$ are orthogonal to $\mathfrak{h}_{\mathfrak{p}}^{\perp}$, so there exist $S, T \in \mathfrak{t}$ such that $S+\|X\|^{2} B-a X, T-2 b X+$ $\|X\|^{2} Z \in \mathfrak{h}$. Then

$$
\begin{aligned}
\mathfrak{h} \ni & {\left[S+\|X\|^{2} B-a X, T-2 b X+\|X\|^{2} Z\right] } \\
& =-2 b[S, X]+a[T, X]-b\|X\|^{2} X+\|X\|^{4} Z .
\end{aligned}
$$

Taking inner product of this vector with $a B+(1-\theta) X+b(1-\theta) Z \in \mathfrak{h}_{\mathfrak{p}}^{\perp}$, and using that the elements of $\operatorname{ad}(\mathfrak{t})$ are skew-symmetric, together with $\langle Z, Z\rangle=2$, yields $0=-b\|X\|^{4}+2 b\|X\|^{4}=b\|X\|^{4}$, which implies $X=0$.

Now take $W \in \mathfrak{w}$. Then $J W$ is orthogonal to $\mathfrak{h}_{\mathfrak{p}}^{\perp}$ and thus there exists $R \in \mathfrak{t}$ such that $R+J W \in \mathfrak{h}$. Since $\mathfrak{h}$ is orthogonal to $\left[\mathfrak{h}_{\mathfrak{p}}^{\perp}, \mathfrak{h}_{\mathfrak{p}}^{\perp}\right]$, we obtain 
using (2.2),

$$
\begin{aligned}
0 & =\langle R+J W,[a B+b(1-\theta) Z,(1-\theta) W]\rangle \\
& =\left\langle R+J W, \frac{a}{2}(1+\theta) W-b(1+\theta) J W\right\rangle=-b\|W\|^{2} .
\end{aligned}
$$

Since $W \in \mathfrak{w}$ is arbitrary we conclude that $\mathfrak{w}=0$. Then we can take $U \in \mathfrak{g}_{\alpha}$ and $S, T \in \mathfrak{t}$ such that $S+U, T+J U \in \mathfrak{h}$. Since $\mathfrak{h}$ is orthogonal to $\mathfrak{h}_{\mathfrak{p}}^{\perp}$, we obtain

$$
\begin{aligned}
0 & =\langle[S+U, T+J U], a B+b(1-\theta) Z\rangle \\
& =\left\langle[S, J U]-[T, U]+\frac{1}{2}\|U\|^{2} Z, a B+b(1-\theta) Z\right\rangle=b\|U\|^{2},
\end{aligned}
$$

so $U=0$. This implies $\mathfrak{g}_{\alpha}=0$, which is not possible in $\mathbb{C} H^{n}, n \geq 2$. Therefore $b=0$.

Let us denote by $(\cdot)_{\mathfrak{a} \oplus \mathfrak{n}}$ and $(\cdot)_{\mathfrak{t}}$ the orthogonal projections onto $\mathfrak{a} \oplus \mathfrak{n}$ and $\mathfrak{t}$, respectively. The next step of our proof is:

Proposition 4.4. There exists an isometry $g \in G$ such that $(\operatorname{Ad}(g) \mathfrak{h})_{\mathfrak{a} \oplus \mathfrak{n}}=$ $\mathfrak{s}_{V, \mathfrak{v}}$ for some subspace $V$ of $\mathfrak{a}$ and some real subspace $\mathfrak{v}$ of $\mathfrak{g}_{\alpha}$.

Proof. Recall that $\mathfrak{h}_{\mathfrak{p}}^{\perp}=\mathbb{R}(a B+(1-\theta) X) \oplus(1-\theta) \mathfrak{w}$, where $a \in \mathbb{R}, X \in$ $\mathfrak{g}_{\alpha} \ominus \mathfrak{w}$, and $\mathbb{R} X \oplus \mathfrak{w}$ is a real subspace of $\mathfrak{g}_{\alpha}$.

If $a=0$ the conclusion is obvious with $g=1$, the identity isometry in $G$, $V=\mathfrak{a}$ and $\mathfrak{v}=\mathbb{R} X+\mathfrak{w}$. So assume $a \neq 0$ and renormalize $X$ so that $a=1$. If $X=0$, the result is again obvious just by taking $g=1$ and $\mathfrak{v}=\mathfrak{w}$, so we will also assume $X \neq 0$.

Under these circumstances, we can define the isometry

$$
g=\exp \left(-\frac{2}{\|X\|^{2}} X\right)
$$

We will show that $(\operatorname{Ad}(g) \mathfrak{h})_{\mathfrak{a} \oplus \mathfrak{n}}=\mathfrak{s}_{\mathfrak{a}, \mathfrak{v}}$, where $\mathfrak{v}=\mathbb{R} X \oplus \mathfrak{w}$. Note that $\mathfrak{h} \cap \mathfrak{k}=$ $\mathfrak{h} \cap \mathfrak{t}$ and $\operatorname{Ad}(g)(\mathfrak{h}) \cap \mathfrak{k}=\operatorname{Ad}(g)(\mathfrak{h}) \cap \mathfrak{t}$ are conjugate because $H$ and $I_{g}(H)$ are conjugate and all their orbits are principal. Since $\operatorname{dim}(\operatorname{Ad}(g) \mathfrak{h})_{\mathfrak{a} \oplus \mathfrak{n}}=$ $\operatorname{dim} \operatorname{Ad}(g) \mathfrak{h}-\operatorname{dim}(\operatorname{Ad}(g)(\mathfrak{h}) \cap \mathfrak{t})=\operatorname{dim} \mathfrak{h}-\operatorname{dim}(\mathfrak{h} \cap \mathfrak{t})=\operatorname{dim} \mathfrak{h}_{\mathfrak{a} \oplus \mathfrak{n}}=\operatorname{dim} \mathfrak{s}_{\mathfrak{a}, \mathfrak{v}}$, it suffices to show that $(\operatorname{Ad}(g) \mathfrak{h})_{\mathfrak{a} \oplus \mathfrak{n}} \subset \mathfrak{s}_{\mathfrak{a}, \mathfrak{v}}$. 
From the definition of $\mathfrak{h}_{\mathfrak{p}}^{\perp}$ it follows that

$$
\mathfrak{h}_{\mathfrak{a} \oplus \mathfrak{n}}=\mathbb{R}\left(\|X\|^{2} B-X\right) \oplus\left(\mathfrak{g}_{\alpha} \ominus \mathfrak{v}\right) \oplus \mathfrak{g}_{2 \alpha}
$$

As in Case 1, all the orbits are principal and $\mathfrak{h} \cap \mathfrak{t}=\mathfrak{h} \cap \mathfrak{k}$ is the isotropy subalgebra of $\mathfrak{h}$ at $o$. Thus we obtain $\left[\mathfrak{h} \cap \mathfrak{t}, \mathfrak{h}_{\mathfrak{p}}^{\perp}\right]=0$, which implies $[\mathfrak{h} \cap \mathfrak{t}, a B+(1-\theta) X]=0$. From this we conclude $[\mathfrak{h} \cap \mathfrak{t}, X]=0$ and $(\operatorname{Ad}(g)(\mathfrak{h} \cap \mathfrak{t}))_{\mathfrak{a} \oplus \mathfrak{n}}=0$.

Let $S \in \mathfrak{t}$ be such that $S+\|X\|^{2} B-X \in \mathfrak{h}$. For any $U \in \mathfrak{g}_{\alpha} \ominus \mathfrak{v}$ there exists $T_{U} \in \mathfrak{t}$ such that $T_{U}+U \in \mathfrak{h}$. Then, $0=\left\langle\left[S+\|X\|^{2} B-X, T_{U}+U\right]\right.$, $B+(1-\theta) X\rangle=\langle[S, U], X\rangle$, so $[S, X] \in \mathfrak{w}$. On the other hand, by (2.3),

$$
\begin{aligned}
0 & =\left\langle S+\|X\|^{2} B-X,[B+(1-\theta) X,(1-\theta) W]\right\rangle=-\langle S,(1+\theta)[\theta X, W]\rangle \\
& =-2\langle[S, X], W\rangle
\end{aligned}
$$

for any $W \in \mathfrak{w}$. Thus, $[S, X]=0$. This implies

$$
\begin{aligned}
\operatorname{Ad}(g)\left(S+\|X\|^{2} B-X\right) & =S+\|X\|^{2} B-X-\frac{2}{\|X\|^{2}}\left(-\frac{\|X\|^{2}}{2} X\right) \\
& =S+\|X\|^{2} B
\end{aligned}
$$

whose projection onto $\mathfrak{a} \oplus \mathfrak{n}$ is $\|X\|^{2} B \in \mathfrak{s} \mathfrak{a}, \mathfrak{v}$.

Now let $U \in \mathfrak{g}_{\alpha} \ominus \mathfrak{v}$ and $T_{U} \in \mathfrak{t}$ such that $T_{U}+U \in \mathfrak{h}$. Clearly, $[X, U],\left[X,\left[X, T_{U}\right]\right] \in \mathfrak{g}_{2 \alpha}$. On the other hand, using (2.3),

$$
\begin{aligned}
0 & =\left\langle T_{U}+U,[B+(1-\theta) X,(1-\theta) W]\right\rangle=-\left\langle T_{U},(1+\theta)[\theta X, W]\right\rangle \\
& =-2\left\langle\left[T_{U}, X\right], W\right\rangle
\end{aligned}
$$

for any $W \in \mathfrak{w}$. This and the skew-symmetry of $\operatorname{ad}\left(T_{U}\right)$ imply $\left[X, T_{U}\right] \in$ $\mathfrak{g}_{\alpha} \ominus \mathfrak{v}$, and thus

$$
\begin{aligned}
& \left(\operatorname{Ad}(g)\left(T_{U}+U\right)\right)_{\mathfrak{a} \oplus \mathfrak{n}} \\
& \quad=\left(T_{U}+U-\frac{2}{\|X\|^{2}}\left[X, T_{U}\right]-\frac{2}{\|X\|^{2}}[X, U]+\frac{2}{\|X\|^{4}}\left[X,\left[X, T_{U}\right]\right]\right)_{\mathfrak{a} \oplus \mathfrak{n}} \\
& \quad=U-\frac{2}{\|X\|^{2}}\left[X, T_{U}\right]-\frac{2}{\|X\|^{2}}[X, U]+\frac{2}{\|X\|^{4}}\left[X,\left[X, T_{U}\right]\right] \\
& \quad \in\left(\mathfrak{g}_{\alpha} \ominus \mathfrak{v}\right) \oplus \mathfrak{g}_{2 \alpha} \subset \mathfrak{s}_{\mathfrak{a}, \mathfrak{v}} .
\end{aligned}
$$

Finally, let $T_{Z} \in \mathfrak{t}$ such that $T_{Z}+Z \in \mathfrak{h}$. Using (2.3) we obtain

$$
0=\left\langle T_{Z}+Z,[B+(1-\theta) X,(1-\theta) W]\right\rangle=-2\left\langle\left[T_{Z}, X\right], W\right\rangle
$$


for any $W \in \mathfrak{w}$, thus showing that $\left[T_{Z}, X\right] \in \mathfrak{g}_{\alpha} \ominus \mathfrak{v}$. Since $\left[X,\left[X, T_{Z}\right]\right] \in \mathfrak{g}_{2 \alpha}$ we obtain

$$
\begin{gathered}
\left(\operatorname{Ad}(g)\left(T_{Z}+Z\right)\right)_{\mathfrak{a} \oplus \mathfrak{n}}=\left(T_{Z}+Z-\frac{2}{\|X\|^{2}}\left[X, T_{Z}\right]+\frac{2}{\|X\|^{4}}\left[X,\left[X, T_{Z}\right]\right]\right)_{\mathfrak{a} \oplus \mathfrak{n}} \\
=Z-\frac{2}{\|X\|^{2}}\left[X, T_{Z}\right]+\frac{2}{\|X\|^{4}}\left[X,\left[X, T_{Z}\right]\right] \in\left(\mathfrak{g}_{\alpha} \ominus \mathfrak{v}\right) \oplus \mathfrak{g}_{2 \alpha} \subset \mathfrak{s}_{\mathfrak{a}, \mathfrak{v}} .
\end{gathered}
$$

Altogether this implies $(\operatorname{Ad}(g) \mathfrak{h})_{\mathfrak{a} \oplus \mathfrak{n}} \subset \mathfrak{s}_{\mathfrak{a}, \mathfrak{w}}$, and since the dimensions are the same, equality follows as we had claimed.

So far we have shown that a group $H$ which induces a polar foliation of $\mathbb{C} H^{n}, n \geq 2$, has a Lie subalgebra $\mathfrak{h}$ that can be assumed to be contained in a maximally noncompact Borel subalgebra $\mathfrak{t} \oplus \mathfrak{a} \oplus \mathfrak{n}$ in such a way that its projection onto $\mathfrak{a} \oplus \mathfrak{n}$ satisfies $\mathfrak{h}_{\mathfrak{a} \oplus \mathfrak{n}}=\mathfrak{s}_{V, \mathfrak{w}}$, where $\mathfrak{s}_{V, \mathfrak{w}}$ is one of our model examples with $V \subset \mathfrak{a}$ a linear subspace, and $\mathfrak{w} \subset \mathfrak{g}_{\alpha}$ a real subspace. Our aim in what follows is to show that the actions of $H$ and $S_{V, \mathfrak{w}}$ are orbit equivalent. The fundamental part of the proof of this fact is contained in the following assertion:

Proposition 4.5. With the notations as above, let $\mathfrak{h}_{\mathfrak{t}}$ denote the orthogonal projection of $\mathfrak{h}$ onto $\mathfrak{t}$. Then $\left[\mathfrak{h}_{\mathfrak{t}}, \mathfrak{w}\right]=0$. In particular, $\mathfrak{h}_{\mathfrak{t}}$ normalizes $V \oplus$ $(\mathfrak{n} \ominus \mathfrak{w})$.

Proof. We begin with some general observations regarding $\mathfrak{h}$. Let $W_{1}, W_{2} \in$ $\mathfrak{w}, U \in \mathfrak{s}_{V, \mathfrak{w}}=V \oplus\left(\mathfrak{g}_{\alpha} \ominus \mathfrak{w}\right) \oplus \mathfrak{g}_{2 \alpha}$, and $T \in \mathfrak{t}$ such that $T+U \in \mathfrak{h}$. Since $\mathfrak{h}$ is orthogonal to $\left[\mathfrak{h}_{\mathfrak{p}}^{\perp}, \mathfrak{h}_{\mathfrak{p}}^{\perp}\right]$ we have by $(2.3)$,

$$
\begin{aligned}
0 & =\left\langle T+U,\left[(1-\theta) W_{1},(1-\theta) W_{2}\right]\right\rangle=-\left\langle T,(1+\theta)\left[\theta W_{1}, W_{2}\right]\right\rangle \\
& =-2\left\langle\left[T, W_{1}\right], W_{2}\right\rangle .
\end{aligned}
$$

This already implies $\left[\mathfrak{h}_{\mathfrak{t}}, \mathfrak{w}\right] \subset \mathfrak{g}_{\alpha} \ominus \mathfrak{w}$.

The fact that all the orbits are principal and that $\mathfrak{h} \cap \mathfrak{t}=\mathfrak{h} \cap \mathfrak{k}$ is the isotropy algebra at $o$, implies $0=\left[\mathfrak{h} \cap \mathfrak{t}, \mathfrak{h}_{\mathfrak{p}}^{\perp}\right]=[\mathfrak{h} \cap \mathfrak{t}, \mathfrak{a} \oplus(1-\theta) \mathfrak{w}]$. Hence we obtain $[\mathfrak{h} \cap \mathfrak{t}, \mathfrak{w}]=0$.

For $W \in \mathfrak{w}$ we define the map $F_{W}: \mathfrak{s}_{V, \mathfrak{w}} \rightarrow \mathfrak{g}_{\alpha} \ominus \mathfrak{w}, U \mapsto\left[W, T_{U}\right]$, where $T_{U} \in \mathfrak{t}$ is any vector satisfying $T_{U}+U \in \mathfrak{h}$. This map is well-defined because given $S_{U}, T_{U} \in \mathfrak{t}$ such that $S_{U}+U, T_{U}+U \in \mathfrak{h}$, we have $S_{U}-T_{U}=$ $\left(S_{U}+U\right)-\left(T_{U}+U\right) \in \mathfrak{h} \cap \mathfrak{t}$, and from $[\mathfrak{h} \cap \mathfrak{t}, \mathfrak{w}]=0$ it follows that $\left[W, S_{U}\right]=\left[W, T_{U}\right]$. The map is clearly linear, and its image is contained in 
$\mathfrak{g}_{\alpha} \ominus \mathfrak{w}$ because $\left[\mathfrak{h}_{\mathfrak{t}}, \mathfrak{w}\right] \subset \mathfrak{g}_{\alpha} \ominus \mathfrak{w}$. The assertion will follow if we show that $F_{W}=0$ for any $W \in \mathfrak{w}$.

Let $H \in V, X \in \mathfrak{g}_{\alpha} \ominus \mathfrak{w}, b \in \mathbb{R}$, and $S, T \in \mathfrak{t}$ such that $T+H+b Z$, $S+X \in \mathfrak{h}$. Since $\left[\mathfrak{t}, \mathfrak{a} \oplus \mathfrak{g}_{2 \alpha}\right]=0$, we obtain $[T, X]+\frac{1}{2}\langle H, B\rangle X=$ $[T+H+b Z, S+X] \in \mathfrak{h}$. Thus, for any $W \in \mathfrak{w}$ we have

$$
0=\left\langle[T, X]+\frac{1}{2}\langle H, B\rangle X,(1-\theta) W\right\rangle=\langle[W, T], X\rangle .
$$

Since $\quad X \in \mathfrak{g}_{\alpha} \ominus \mathfrak{w} \quad$ is arbitrary and $\quad\left[\mathfrak{h}_{\mathfrak{t}}, \mathfrak{w}\right] \subset \mathfrak{g}_{\alpha} \ominus \mathfrak{w}$, we obtain $F_{W}\left(V \oplus \mathfrak{g}_{2 \alpha}\right)=0$ for any $W \in \mathfrak{w}$.

Let us denote by $\tilde{F}_{W}: \mathfrak{g}_{\alpha} \ominus \mathfrak{w} \rightarrow \mathfrak{g}_{\alpha} \ominus \mathfrak{w}$ the restriction of $F_{W}$ to $\mathfrak{g}_{\alpha} \ominus \mathfrak{w}$. It now suffices to prove that $\tilde{F}_{W}=0$ for any $W \in \mathfrak{w}$.

Let $X, Y \in \mathfrak{g}_{\alpha} \ominus \mathfrak{w}$ and $T_{X}, T_{Y} \in \mathfrak{t}$ be such that $T_{X}+X, T_{Y}+Y \in \mathfrak{h}$. Then $\left[T_{X}, Y\right]-\left[T_{Y}, X\right]+\frac{1}{2}\langle J X, Y\rangle Z=\left[T_{X}+X, T_{Y}+Y\right] \in \mathfrak{h}$, and thus, for any $W \in \mathfrak{w}$, taking inner product with $(1-\theta) W$, we obtain $\left\langle\left[W, T_{X}\right], Y\right\rangle=$ $\left\langle\left[W, T_{Y}\right], X\right\rangle$. This readily implies $\left\langle\tilde{F}_{W}(X), Y\right\rangle=\left\langle\tilde{F}_{W}(Y), X\right\rangle$ for any $X, Y \in$ $\mathfrak{g}_{\alpha} \ominus \mathfrak{w}$, so $\tilde{F}_{W}$ is a self-adjoint endomorphism of $\mathfrak{g}_{\alpha} \ominus \mathfrak{w}$, and hence, it is diagonalizable with real eigenvalues.

Assume, by contradiction, that there exists $W \in \mathfrak{w}$ such that $\tilde{F}_{W} \neq$ 0 . Then there is an eigenvalue $\lambda \neq 0$ of $\tilde{F}_{W}$ and a nonzero vector $X \in$ $\mathfrak{g}_{\alpha} \ominus \mathfrak{w}$ such that $\tilde{F}_{W}(X)=\lambda X$. Let $T_{X} \in \mathfrak{t}$ be such that $T_{X}+X \in \mathfrak{h}$; then $\left[W, T_{X}\right] \neq 0$ because $\tilde{F}_{W}(X) \neq 0$.

We define $g=\exp \left(-\frac{1}{\lambda} W\right)$. The groups $H$ and $I_{g}(H)$ are conjugate, and since $H$ induces a foliation so does $I_{g}(H)$. Since all the orbits of a homogeneous foliation are principal and $H$ and $I_{g}(H)$ are conjugate, so are their isotropy groups at the origin, $H_{o}$ and $I_{g}(H)_{o}$ respectively. In particular, their Lie algebras must have the same dimension, that is, $\operatorname{dim}(\mathfrak{h} \cap \mathfrak{k})=$ $\operatorname{dim}(\operatorname{Ad}(g)(\mathfrak{h}) \cap \mathfrak{k})$. We will see that this is not true, hence obtaining our contradiction.

Clearly, $\mathfrak{h} \cap \mathfrak{k}=\mathfrak{h} \cap \mathfrak{t}$. On the other hand, since $[\mathfrak{h} \cap \mathfrak{t}, \mathfrak{w}]=0$, we obtain $\operatorname{Ad}(g) \mid(\mathfrak{h} \cap \mathfrak{t})=1_{\mathfrak{h} \cap \mathfrak{t}}$, the identity on $\mathfrak{h} \cap \mathfrak{t}$, so $\mathfrak{h} \cap \mathfrak{t} \subset \operatorname{Ad}(g)(\mathfrak{h}) \cap \mathfrak{k}$. Finally, let $b=\frac{1}{2 \lambda}\langle J W, X\rangle-\frac{1}{4 \lambda^{2}}\left\langle J W,\left[W, T_{X}\right]\right\rangle$ and $S \in \mathfrak{t}$ such that $S+b Z \in \mathfrak{h}$. Recall that $[W, S]=0$ because of $F_{W}\left(\mathfrak{g}_{2 \alpha}\right)=0$. Then,

$$
\begin{aligned}
& \operatorname{Ad}(g)\left(T_{X}+X+S+b Z\right) \\
& \quad=\sum_{k=0}^{\infty} \frac{1}{k !} \operatorname{ad}\left(-\frac{1}{\lambda} W\right)^{k}\left(T_{X}+X+S+b Z\right) \\
& \quad=T_{X}+X+S+b Z-\frac{1}{\lambda}\left(\left[W, T_{X}\right]+[W, X]\right)+\frac{1}{2 \lambda^{2}}\left[W,\left[W, T_{X}\right]\right]
\end{aligned}
$$




$$
\begin{aligned}
& =T_{X}+S+X-\frac{1}{\lambda} \tilde{F}_{W}(X)+\left(b-\frac{1}{2 \lambda}\langle J W, X\rangle+\frac{1}{4 \lambda^{2}}\left\langle J W,\left[W, T_{X}\right]\right\rangle\right) Z \\
& =T_{X}+S \in \mathfrak{k} .
\end{aligned}
$$

Now, $T_{X}+S \notin \mathfrak{t} \cap \mathfrak{h}$ as $\left[W, T_{X}\right] \neq 0,[S, \mathfrak{w}]=0$, and $[\mathfrak{h} \cap \mathfrak{t}, \mathfrak{w}]=0$. This exhibits the fact that $\mathfrak{h} \cap \mathfrak{t}$ is strictly contained in $\operatorname{Ad}(g)(\mathfrak{h}) \cap \mathfrak{k}$, which contradicts the property that $H$ induces a foliation. Therefore $\tilde{F}_{W}=0$ for all $W \in \mathfrak{w}$, and we obtain $\left[\mathfrak{h}_{\mathfrak{t}}, \mathfrak{w}\right]=0$.

In order to obtain the last assertion, first observe that $\mathfrak{t}$ centralizes $\mathfrak{a} \oplus$ $\mathfrak{g}_{2 \alpha}$. Now if $X \in \mathfrak{g}_{\alpha}, T \in \mathfrak{h}_{\mathfrak{t}}$, and $W \in \mathfrak{w}$, we obtain $\langle[T, X], W\rangle=$ $-\langle[T, W], X\rangle=0$, from where it follows that $\mathfrak{h}_{\mathfrak{t}}$ normalizes $\mathfrak{g}_{\alpha} \ominus \mathfrak{w}$.

Now we are ready for the final step of the proof.

Proposition 4.6. Let $\mathfrak{t} \oplus \mathfrak{a} \oplus \mathfrak{n}$ be a maximally noncompact Borel subalgebra of $\mathfrak{g}$. Let $\mathfrak{h}$ be a subalgebra of $\mathfrak{t} \oplus \mathfrak{a} \oplus \mathfrak{n}$ such that $\mathfrak{h}_{\mathfrak{a} \oplus \mathfrak{n}}=\mathfrak{s}_{V, \mathfrak{w}}$. Assume that the orbits of the connected subgroup $H$ of $G$ whose Lie algebra is $\mathfrak{h}$ form a homogeneous polar foliation of $\mathbb{C} H^{n}$. Then the actions of $H$ and $S_{V, \mathfrak{w}}$ on $\mathbb{C} H^{n}$ are orbit equivalent.

Proof. First recall from Proposition 4.5 that $\mathfrak{h}_{\mathfrak{t}}$ normalizes $V \oplus\left(\mathfrak{g}_{\alpha} \ominus \mathfrak{w}\right) \oplus$ $\mathfrak{g}_{2 \alpha}=V \oplus(\mathfrak{n} \ominus \mathfrak{w})$. We also denote by $H_{\mathfrak{t}}$ the connected subgroup of $S U(1, n)$ whose Lie algebra is $\mathfrak{h}_{\mathfrak{t}}$.

We now show that an element $g \in H$ sufficiently close to the identity in $H$ can be written as $g=\tan$ where $t \in H_{\mathfrak{t}}, a \in \exp (V) \subset A$ and $n \in S_{\{0\}, \mathfrak{w}}$, where as usual $S_{\{0\}, \mathfrak{w}}$ is the connected subgroup of $A N$ whose Lie algebra is $\mathfrak{n} \ominus \mathfrak{w}$. First write $g=\exp (T+a B+X)$ with $T \in \mathfrak{t}, a \in \mathbb{R}$, and $X \in \mathfrak{n} \ominus \mathfrak{w}$. By the Iwasawa decomposition we can write $g=\exp (S) \exp (b B) \exp (Y)$, with $S \in \mathfrak{t}, b \in \mathbb{R}$, and $Y \in \mathfrak{n}$. The Baker-Campbell-Hausdorff formula yields

$$
\begin{aligned}
\exp (Y) & =\exp (-b B) \exp (-S) \exp (T+a B+X) \\
& =\exp (-S-b B) \exp (T+a B+X) \\
& =\exp \left(T-S+(a-b) B+X-\frac{1}{2}[S, X]-\frac{b}{2}[B, X]+\cdots\right),
\end{aligned}
$$

where the dots involve linear combinations of iterated brackets of $X$ with $S$ and $B$. Comparing both sides of the equation we immediately obtain $S=T$ and $b=a$. Now, since $\mathfrak{h}_{\mathfrak{t}} \oplus \mathfrak{a}$ normalizes $\mathfrak{n} \ominus \mathfrak{w}$, the right-hand side of the equation above is in $\mathfrak{n} \ominus \mathfrak{w}$, and so is $Y$, as we wanted to show.

From Proposition 4.5 we also have $\left[\mathfrak{h}_{\mathfrak{t}}, \mathfrak{w}\right]=0$, which obviously implies $\operatorname{Ad}(\exp (T)) \xi=\xi$ for any $\xi \in(1-\theta) \mathfrak{w}$ and $T \in \mathfrak{h}_{\mathfrak{t}}$. Let $g \in H$ be sufficiently close to the identity of $H$ and write $g^{-1}=\tan$ with $t \in H_{\mathfrak{t}} \subset K, a \in$ 
$\exp (V) \subset A$ and $n \in S_{\{0\}, \mathfrak{w}}$ as explained above. Then $g=n^{-1} a^{-1} t^{-1}$, and since $A$ normalizes $S_{\{0\}, \mathfrak{w}}$ we can write $n^{-1} a^{-1}=a^{-1} n^{\prime}$ with $n^{\prime} \in S_{\{0\}, \mathfrak{w}}$. Thus, $g(o)=a^{-1} n^{\prime} t^{-1}(o)=a^{-1} n^{\prime}(o)$ and hence $H \cdot o \subset S_{V, \mathfrak{w}} \cdot o$ in a neighborhood of $o \in H \cdot o$. Since both orbits $H \cdot o$ and $S_{V, \mathfrak{w}} \cdot o$ have the same dimension and are connected and complete we conclude $H \cdot o=S_{V, \mathfrak{w}} \cdot o$.

Now, let $p=\exp _{o}(\xi)$ with $\xi \in \nu_{o}(H \cdot o) \cong(\mathfrak{a} \ominus V) \oplus(1-\theta) \mathfrak{w} \subset \mathfrak{p}$. Using the fact that $H$ acts isometrically on $\mathbb{C} H^{n}, n \geq 2$, and that $t_{*}^{-1} \xi=\operatorname{Ad}\left(t^{-1}\right)$ $\xi=\xi$ we obtain

$$
\begin{aligned}
g(p) & =\exp _{g(o)}\left(g_{*} \xi\right)=\exp _{a^{-1} n^{\prime} t^{-1}(o)}\left(\left(a^{-1} n^{\prime} t^{-1}\right)_{*} \xi\right) \\
& =\exp _{a^{-1} n^{\prime}(o)}\left(\left(a^{-1} n^{\prime}\right)_{*} \xi\right)=a^{-1} n^{\prime}(p)
\end{aligned}
$$

Hence, $H \cdot p \subset S_{V, \mathfrak{w}} \cdot p$ in a neighborhood of $p \in H \cdot p$, and as above we can conclude that both orbits coincide. Since the action of $H$ is polar, all the orbits can be obtained in this way, and so, $H$ and $S_{V, \mathfrak{w}}$ have the same orbits.

This concludes the proof of the Main Theorem.

\section{Acknowledgments}

The authors would like to thank the referee for valuable comments which improved some arguments in the original version of this article. The second author has been supported by a Marie-Curie European Reintegration Grant (no. PERG04-GA-2008-239162) and projects MTM2009-07756 and INCITE09207151PR (Spain)

\section{References}

[1] J. Berndt, J.C. Díaz-Ramos and H. Tamaru, Hyperpolar homogeneous foliations on symmetric spaces of noncompact type, J. Differential Geom. 86 (2010), 191-235.

[2] J. Berndt and H. Tamaru, Homogeneous codimension one foliations on noncompact symmetric spaces, J. Differential Geom. 63 (2003), 1-40.

[3] J. Berndt and H. Tamaru, Cohomogeneity one actions on symmetric spaces of noncompact type, preprint arXiv:1006.1980v1, to appear in J. Reine Angew. Math. Available at: http://www.degruyter.com/ view/j/crelle.ahead-of-print/crelle-2012-0002/crelle-2012-0002.xml? format $=\mathrm{INT}$ 
[4] J. Berndt, F. Tricerri and L. Vanhecke, Generalized Heisenberg groups and Damek-Ricci harmonic spaces, Lecture Notes in Mathematics, Springer-Verlag, Berlin, 1598, 1995.

[5] J. Dadok, Polar coordinates induced by actions of compact Lie groups, Trans. Amer. Math. Soc. 288 (1985), 125-137.

[6] J.C. Díaz-Ramos, A. Kollross, Polar actions with a fixed point, Differential Geom. Appl. 29 (2011), 20-25.

[7] C. Gorodski, Polar actions on compact symmetric spaces which admit a totally geodesic principal orbit, Geom. Dedicata 103 (2004), 193-204.

[8] A.W. Knapp, Lie groups beyond an introduction, Birkhäuser, Boston MA, 1996.

[9] A. Kollross, Polar actions on symmetric spaces, J. Differential Geom. 77 (2007), 425-482.

[10] A. Kollross, Low cohomogeneity and polar actions on exceptional compact Lie groups, Transf. Groups 14 (2009), 387-415.

[11] A. Kollross, Duality of symmetric spaces and polar actions, J. Lie Theory 21 (2011), 961-986.

[12] G.D. Mostow, On maximal subgroups of real Lie groups, Ann. of Math. $\mathbf{7 4}(2)$ (1961), 503-517.

[13] F. Podestà and G. Thorbergsson, Polar actions on rank-one symmetric spaces, J. Differential Geom. 53 (1999), 131-175.

[14] B. Wu, Isoparametric submanifolds of hyperbolic spaces, Trans. Amer. Math. Soc. 331 (1992), 609-626.

Department of Mathematics

King's COLLEGE LONDON

LONDON, WC2R 2LS

$\mathrm{UK}$

E-mail address: jurgen.berndt@kcl.ac.uk

Department of Geometry and Topology

FACULTY OF MATHEMATICS

Av. Lope Gomez de Marzoa s/N

University of SANTiago de Compostela

15786 Santiago de Compostela

SPAIN

E-mail address: josecarlos.diaz@usc.es

Received November 11, 2011 